\title{
Targeted treatments in advanced renal cell carcinoma: focus on axitinib
}

This article was published in the following Dove Press journal:

Pharmacogenomics and Personalized Medicine

27 March 2014

Number of times this article has been viewed

\section{Elena Verzoni \\ Paolo Grassi \\ Isabella Testa \\ Roberto lacovelli \\ Pamela Biondani \\ Enrico Garanzini \\ Filippo De Braud \\ Giuseppe Procopio}

Department of Medical Oncology I, Fondazione IRCCS Istituto Nazionale Tumori, Milan, Italy
Correspondence: Giuseppe Procopio Department of Medical Oncology I, Fondazione IRCCS Istituto Nazionale Tumori, Via G Venezian I, 20I33 Milan, Italy

Tel +3902 23904450

Fax +390223902149

Email giuseppe.procopio@istitutotumori. mi.it

\begin{abstract}
Antiangiogenesis options have evolved rapidly in the last few years, with an increasing number of agents currently approved by the US Food and Drug Administration and European Medicines Agency. Angiogenesis inhibitors have been shown to be very effective for the treatment of metastatic renal cancer cell. Axitinib is a third-generation inhibitor of vascular endothelial growth factor receptor and is currently being developed for the treatment of various malignancies. The pharmacokinetic properties of axitinib may have a selective therapeutic effect, with minimal adverse reactions and enhanced safety. In a large Phase III study of previously treated patients with metastatic renal cell carcinoma, axitinib achieved a longer progression-free survival than sorafenib with an acceptable safety profile and good quality of life. This review focuses on the pharmacology, pharmacokinetics, and clinical activity of axitinib in the current treatment of renal cell carcinoma. The role of axitinib in the adjuvant and/or neoadjuvant setting needs to be evaluated in further clinical trials.
\end{abstract}

Keywords: axitinib, renal cell carcinoma, vascular endothelial growth factor receptor, angiogenesis

\section{Introduction}

Renal cell carcinoma (RCC) accounts for almost $85 \%$ of all kidney cancers. ${ }^{1}$ Over 120,000 cases of RCC are currently diagnosed each year in Europe and the USA, ${ }^{2,3}$ and the incidence of RCC appears to be rising. ${ }^{4,5}$ Approximately $30 \%$ of patients present with metastatic disease and $25 \%$ with locally advanced RCC. ${ }^{4} \mathrm{RCC}$ one of the malignancies most resistant to conventional cytotoxic chemotherapy. ${ }^{6}$ Moreover, up to $40 \%-50 \%$ of patients who undergo curative renal resection go on to develop metastatic or advanced RCC. ${ }^{7,8}$ The 5-year survival rates for patients with kidney cancer, according to the American Joint Committee on Cancer staging classification, are 81\% for stage I, 74\% for stage II, 53\% for stage III, and 8\% for stage IV. ${ }^{4}$ According to the Surveillance, Epidemiology, and End Results Program database, the 5-year survival rates are 91.7\% for localized, $64.2 \%$ for regional, $12.3 \%$ for distant, and $33.5 \%$ for unstaged disease. ${ }^{9}$

Before 2005, standard of care was limited to cytokine therapy with interleukin-2 and/or interferon-alpha (IFN- $\alpha$ ). These treatments were associated with limited efficacy and high toxicity, and remained an option for first-line treatment in only a small minority of highly selected patients with a good prognosis. ${ }^{10-12}$

More recently, a better understanding of the molecular mechanisms involved in the pathogenesis of RCC led to treatment options that target angiogenesis by direct inhibition of vascular endothelial growth factor (VEGF)-mediated signaling and by inhibition of the mammalian target of rapamycin (mTOR) that targets downstream 
signaling and tumor metabolism as well. ${ }^{13-15}$ Several of these novel targeted regimens (bevacizumab, IFN- $\alpha$ combination, sorafenib, temsirolimus, sunitinib, pazopanib, everolimus, and axitinib) have subsequently been evaluated in large robust randomized controlled trials conducted in both the first-line and second-line settings. ${ }^{16}$ Data from these clinical trials have showed superior progression-free survival with targeted agents compared with IFN- $\alpha$ and placebo (in the second-line setting). ${ }^{17}$ As a result of the efficacy results and the US and European regulatory approval for several of these agents, many organizations have updated their clinical practice guidelines. ${ }^{18,19}$ Despite the proliferation of targeted agents approved by the US Food and Drug Administration, RCC continues to have a deleterious impact. In 2012, RCC was the seventh most common malignancy in the US, with an estimated new 65,150 cases and 13,680 deaths in $2013 .{ }^{5}$

Multiple novel therapies targeting the mTOR and VEGF signaling pathways have been introduced into clinical practice, resulting in significant improvements in outcomes for patients with metastatic RCC, compared with cytokine-based therapy, ie, the previous standard of care. Therapies currently approved in the USA and European Union for treatment of patients with metastatic RCC targeting the VEGF pathway include bevacizumab (plus IFN- $\alpha$ ), a humanized monoclonal antibody that inhibits binding of VEGF to its receptor, and the multitargeted receptor tyrosine kinase inhibitors (TKIs) sunitinib, sorafenib, and pazopanib. ${ }^{20-24}$ These agents have diverse molecular profiles and different affinities for VEGF and platelet-derived growth factor receptors, inhibiting some but not all proangiogenic receptors. ${ }^{25}$ The mTOR inhibitors currently approved for treating patients with metastatic RCC are everolimus and temsirolimus. ${ }^{26,27}$

Axitinib (Inlyta ${ }^{\circledR}$; Pfizer Inc., New York, NY, USA) is a third-generation VEGF receptor inhibitor that is highly selective and potent for VEGF receptors 1, 2, and 3 at clinically achievable doses compared with many other antiangiogenic agents in its class. Based on its efficacy in terms of progression-free survival compared with sorafenib in the second-line setting (AXIS [axitinib versus sorafenib in advanced renal cell carcinoma] trial), ${ }^{28}$ in 2012 both the US Food and Drug Administration ${ }^{29}$ and the European Medicines Agency (report EMA/CHMP/453325/2012) approved axitinib for use in the second-line treatment of patients with metastatic RCC after failure of prior treatment with sunitinib or a cytokine.

\section{Pharmacology/mode of action}

Axitinib (AG-013736) is a substituted indazole chemically known as N-methyl-2-[3-(E)-2-pyridin-2yl-vinyl-1H-indazol-6-ylsulfanyl] benzamide, having the formula $\mathrm{C}_{22} \mathrm{H}_{18} \mathrm{~N}_{4} \mathrm{OS}$ and a molecular weight of 386.47 Da (Figure 1). Its synthesis is described under US Patent 6,534,524. ${ }^{30}$ Axitinib is a third-generation, smallmolecule, competitive inhibitor binding to the ATP-binding domain of VEGF receptor 1, 2, and 3 TKIs. Inhibition occurs at nanomolar concentrations and is equally selective for VEGF receptors 1, 2, and 3 compared with other tyrosine kinase receptors, such as c-KIT and platelet-derived growth factor receptor- $\beta$ (Table 1). ${ }^{31}$ In endothelial cells, inhibition of VEGF receptors 1 and 2 by axitinib produces a rapid and dose-dependent reduction in phosphorylation of Akt, endothelial nitric oxide synthase, and extracellular signal-related kinase $1 / 2$. In several in vivo mouse tumor models, axitinib showed antitumor efficacy in association with decreased vascular angiogenesis and increased tumor apoptosis. $^{32}$

\section{Pharmacokinetics}

A Phase I study showed that axitinib is rapidly absorbed when administered with food, with peak plasma concentrations occurring at 2-6 hours following dosing. When dosed in a fed state, peak plasma concentration and area under the plasma concentration-time curve over 24 hours $\left(\mathrm{AUC}_{0-24 \mathrm{~h}}\right)$ are decreased compared with the fasted state (coefficient of variation $90 \%$ and $63 \%$, respectively). Peak concentrations occur 1-2 hours after dosing in the fasted state. Food affects the bioavailability of axitinib; plasma exposure increases by a median of $49 \%$ when axitinib is dosed in the fasted state. Pharmacokinetic assessment suggests a linear dose-proportional relationship between peak plasma concentration and $\mathrm{AUC}_{0-24 \mathrm{~h}}$ (Figure 2). ${ }^{33}$ The terminal

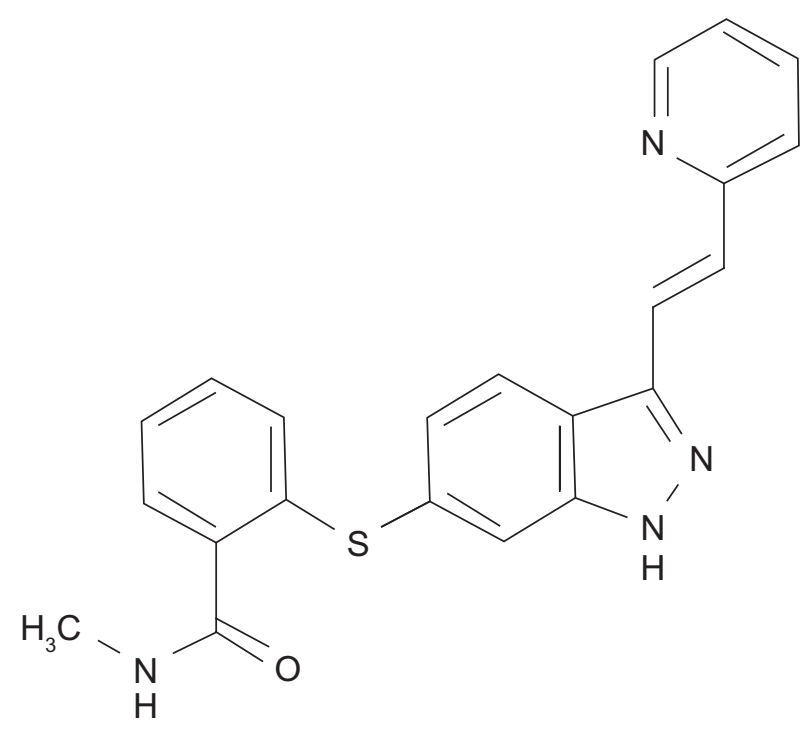

Figure I Chemical structure of axitinib. 
Table I $I C_{50}$ values determined by receptor phosphorylation assays

\begin{tabular}{lll}
\hline Target kinase & $\mathbf{I C}_{\mathbf{5 0}}(\mathrm{nmol} / \mathbf{L})$ & Assayed cells \\
\hline VEGFR-I & 0.06 & HUVEC \\
VEGFR-2 & 0.1 & HUVEC \\
VEGFR-3 & $0.1-0.3$ & PAE \\
PDGFR- $\beta$ & 2.9 & NIH-3T3 \\
\hline
\end{tabular}

Notes: $\mathrm{IC}_{50}$ values were measured by immunoprecipitation/immunoblotting in the presence of $1 \%$ fetal bovine serum (HUVEC) or $0.1 \%$ fetal bovine serum (PAE or $\mathrm{NIH} 3 \mathrm{~T} 3$ ). The data were corrected for protein binding with the consideration that the presence of $2.3 \%$ bovine serum albumin typically shifted cellular $I \mathrm{C}_{50}$ values up by a factor of ten. Reprinted from Clinical Cancer Research, 2008, 14(22), 7272-7283, ${ }^{32}$ Hu-Lowe D, Zou H, Grazzini M, et al, Nonclinical antiangiogenesis and antitumor activities of axitinib (AG-0 13736), an oral, potent, and selective inhibitor of vascular endothelial growth factor receptor tyrosine kinases I, 2, 3, with permission from AACR. Copyright @ 2008, American Association for Cancer Research.

Abbreviations: PAE, porcine aortic endothelial; PDGFR- $\beta$, platelet-derived growth factor receptor-beta; VEGFR, vascular endothelial growth factor receptor; HUVEC, human umbilical vein endothelial cells.

half-life of axitinib is 6-7 hours. ${ }^{34}$ In the Phase I study, it was discovered that the aqueous solubility of AG-013736 is pH-dependent, with low $\mathrm{pH}$ conferring the highest solubility. However, drug concentrations were not significantly affected by the proton pump inhibitor rabeprazole..$^{33}$ Other contributions to absorption of axitinib are the ATP-binding cassette $(\mathrm{ABC})$ transporters P-glycoprotein (ABCB1/MDR-1) and breast cancer resistance protein (BCRP/ABCG2). ${ }^{35}$ These transporters are expressed on the apical membranes of enterocytes, hepatocytes, renal tubular epithelial cells, and brain endothelial capillary cells of the blood-brain barrier, affecting the extent of absorption, secretion, and distribution of certain anticancer drugs across the blood-brain barrier. Transport assay studies and pharmacokinetic studies in

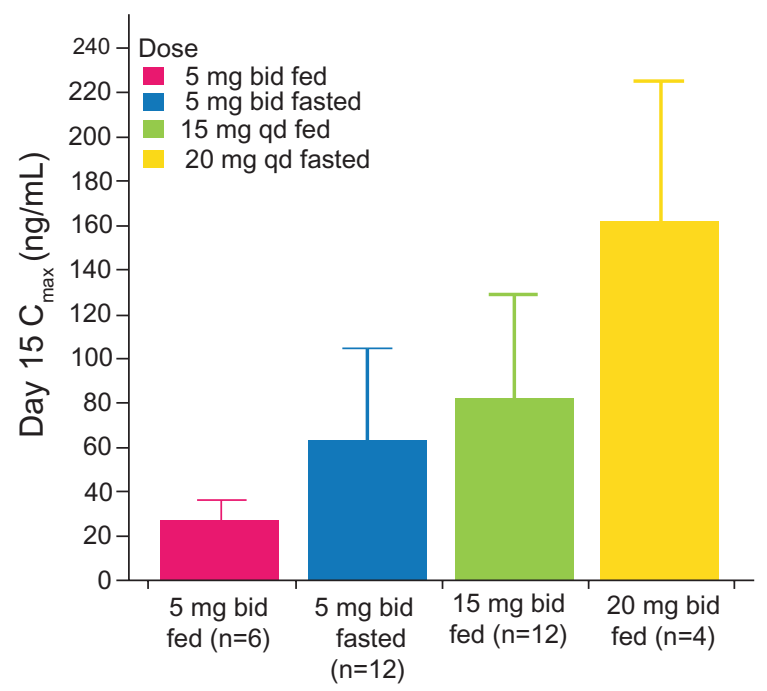

Figure 2 Mean steady-state plasma concentrations on day 15 of dosing (maximum concentration versus dose).

Note: Patson B, Cohen RB, Olszanski AJ, Expert Opinion on Drug Metabolism \& Toxicology, 8(2):259-270..$^{30}$ Copyright (C) 2012, Informa Healthcare. Reproduced with permission of Informa Healthcare.

Abbreviations: bid, twice daily; qd, once daily; $\mathrm{C}_{\max }$, maximum concentration. knockout mice have demonstrated a major role of $\mathrm{ABCB} 1$ in limiting accumulation of axitinib in the brain, compared with $\mathrm{ABCG} 2$ that in turn has an impact on oral bioavailability and plasma axitinib concentrations. ${ }^{35}$ Based on the studies in mice, there is a concern about an increased risk of adverse drug reactions in humans when ABCG2-inhibiting drugs, such as pantoprazole, are coadministered with axitinib, resulting in decreased hepatobiliary excretion or increased gastrointestinal absorption. ${ }^{35}$

The effects of $\mathrm{ABCB} 1$ and $\mathrm{ABCB} 2$ transporters on drug concentrations in brain and plasma are not only important for clinical efficacy but may also be relevant to drug resistance, because ABCB1 (MDR-1) expression correlates with a poorer prognosis in RCC patients. ${ }^{36}$ Further investigation will be needed to elucidate this point fully.

Cytochrome P450 (CYP) subfamily member CYP3A4 accounts for the majority of hepatic metabolism of axitinib, with a smaller proportion being metabolized by CYP1A2, CYP2C19, and glucuronidation. ${ }^{37}$ Two nonactive metabolites are formed, ie, the M7 sulfoxide metabolite, which is a byproduct of the CYP system, and the M12 N-glucuronide metabolite, via the glucuronidation pathway. Ketoconazole, a CYP3A4 inhibitor, significantly increased the peak plasma concentration (by $\sim 1.5$-fold) and $\mathrm{AUC}_{0-24 \mathrm{~h}}$ (by $\sim 2$-fold). ${ }^{37}$

In a Phase I study, rifampin, a potent inducer of the CYP system, decreased the peak plasma concentration and $\mathrm{AUC}_{\text {inf }}$ of axitinib by $71 \%$ and $79 \%$, respectively. Similarly, phenytoin, another potent inducer of multiple CYP enzymes, reduced the $\mathrm{AUC}_{0-24 \mathrm{~h}}$ and peak plasma concentration of AG-013736 by approximately 10 -fold, as observed in a single patient. ${ }^{33}$ Glucuronidation of axitinib occurs via uridine diphosphate glucuronosyl-transferase (UGT)1A1. Patients homozygous for the UGT1A $1 * 28$ variant gene may have increased exposure to drugs metabolized through this pathway, as exemplified by irinotecan. ${ }^{38}$ The pharmacokinetics of axitinib were assessed in four subjects (three Caucasian and one Japanese) known to have the variant UGT1A1*28 allele. In this small population, pharmacokinetic parameters were similar to those lacking the variant, suggesting that patients with the UGT1A1*28 polymorphism probably do not require modification of their axitinib dose. ${ }^{34} \mathrm{~A}$ comprehensive list of CYP3A4 inducers is reported in Table 2.

The fundamental Phase I dose-escalation study in patients with advanced solid tumors dosed patients from $5 \mathrm{mg}$ twice daily to $30 \mathrm{mg}$ twice daily. This study also explored the effects of food on the bioavailability of axitinib, as described earlier. Dose-limiting toxicities were realized at doses of $10 \mathrm{mg}$ twice daily and higher. Two patients with non-small cell adenocarcinoma of the lung initially received $20 \mathrm{mg}$ twice 
Table 2 Main CYP 3A4 inducers

\begin{tabular}{|c|c|}
\hline Inducer & Reference \\
\hline Carbamazepine & 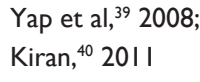 \\
\hline Corticosteroids & Kiran, ${ }^{40} 201 \mathrm{I}$ \\
\hline Efavirenz & Kiran, ${ }^{40} 20 \mathrm{II}$ \\
\hline Felbamate & Yap et al, ${ }^{39} 2008$ \\
\hline Modafinil & Kiran, ${ }^{40} 201 \mathrm{I}$ \\
\hline Nevirapine & Kiran, ${ }^{40} 201 \mathrm{I}$ \\
\hline Omeprazole & Kiran, ${ }^{40} 201 \mathrm{I}$ \\
\hline Oxcarbazepine & 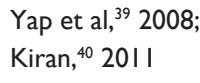 \\
\hline Phenobarbital & Yap et al, ${ }^{39} 2008$ \\
\hline Phenytoin/phosphophenytoin & 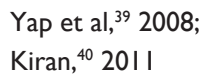 \\
\hline Primidone & 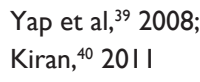 \\
\hline Rifabutin & Kiran, ${ }^{40} 2011$ \\
\hline Rifampin & Kiran, ${ }^{40} 201 \mathrm{I}$ \\
\hline St John's wort (Hypericum perforatum) & Kiran, ${ }^{40} 201 \mathrm{I}$ \\
\hline Topiramate & Yap et al, ${ }^{39} 2008$ \\
\hline
\end{tabular}

daily (subsequently reduced to $10 \mathrm{mg}$ twice daily) and died of fatal hemoptysis, which was considered to be treatmentrelated in one case. Additionally, two patients had hypertensive seizures at respective doses of $20 \mathrm{mg}$ and $10 \mathrm{mg}$ twice daily. ${ }^{33}$ Therefore, the dose of $5 \mathrm{mg}$ twice daily in the fasted state given continuously for a 28-day cycle was selected for subsequent clinical development of axitinib. ${ }^{33}$ Further investigation of axitinib led to the discovery of various crystal forms. Axitinib developed as the polymorph form XLI had a similar pharmacokinetic profile in the fed and fasted state in healthy human volunteers. ${ }^{41}$ The primary toxicity, and a class effect seen across similar VEGF receptor-targeted compounds, was hypertension (all grades), which developed in $61 \%$ of patients. At doses of $5 \mathrm{mg}$ twice daily, hypertension was manageable using standard antihypertensive agents. Other primary toxicities included fatigue, diarrhea, stomatitis, nausea, and vomiting.

\section{Early Phase I and II studies}

The principal evidence for the clinical efficacy of axitinib in subjects with advanced/metastatic RCC is derived from two studies: a Phase II single-arm trial in cytokine-refractory patients; and a multicenter, randomized Phase III study of axitinib as a second-line therapy in patients with metastatic RCC. The single-arm, open-label, multicenter Phase II study of axitinib in subjects with advanced RCC after failure of treatment with one prior cytokine-based therapy ${ }^{42}$ enrolled 52 subjects. Efficacy endpoints included overall response rate (primary endpoint), progression-free survival, time to tumor progression, duration of response, and overall survival. Patients with progressive metastatic RCC were given a starting dose of axitinib $5 \mathrm{mg}$ twice daily in the fasted state. Treatment was continued until no longer of clinical benefit. The patients had a median age of 59 years and were mainly males (77\%). The majority had a previous nephrectomy (94\%), and almost all had tumors with clear-cell histology (98\%; one patient had papillary histology). All patients had received prior treatment with cytokines, the most common of which was IFN alone (52\%). Overall, $58 \%$ of patients had at least one Memorial Sloan Kettering Cancer Center risk factor (ie, at least one poor prognostic factor) for second-line treatment. The investigator-assessed overall response rate was $44.2 \%$ (95\% confidence interval [CI] 30.5-58.7), with two patients achieving a complete response and 21 patients achieving a partial response. Stable disease for 8 weeks or longer was reported in 22 patients (42\%), with 13 patients (25\%) achieving stable disease for 24 weeks or longer. Median response duration was 23.0 months (95\% CI 20.9, not estimable). Maximum percentage decrease in target lesion size, based on Response Evaluation Criteria In Solid Tumors (RECIST) is shown in Figure 3. A decrease of more than 30\% indicates a potential partial response, while a $100 \%$ decrease indicates a potential complete response. The vast majority of patients had some degree of tumor shrinkage $(81 \% ; 42 / 52)$. Only two of the five patients with a $100 \%$ decrease in target lesion size were judged to have a complete response. Median overall survival was 29.9 months ( $95 \%$ CI 20.3 , not estimable) with a median follow-up of 31 months. Median time to progression was 15.7 months (95\% CI 8.4-23.4) and one-year survival was $78.8 \%$ (95\% CI 67.7-89.9).

In the other single-arm, open-label, multicenter Phase II study of axitinib in subjects with advanced RCC after failure of treatment with at least one prior sorafenib-based therapy, most subjects had additionally received prior treatment with sunitinib and/or other agents. ${ }^{43}$ Sixty-two subjects were enrolled and treated. Efficacy endpoints included overall response rate (primary endpoint), progression-free survival, duration of response, and overall survival. Disease response and progression were determined by investigators' assessments. Patients had metastatic RCC refractory to prior therapies that included but were not limited to sorafenib. Axitinib was administered at a starting dose of $5 \mathrm{mg}$ twice daily. Individual dose titrations $>5 \mathrm{mg}$ twice daily and modifications $<5 \mathrm{mg}$ twice daily were permitted, based on individual patient tolerability. At baseline, patients had a median age of 60 (range 35-77) years and 66\% had an Eastern Cooperative Oncology Group performance status 


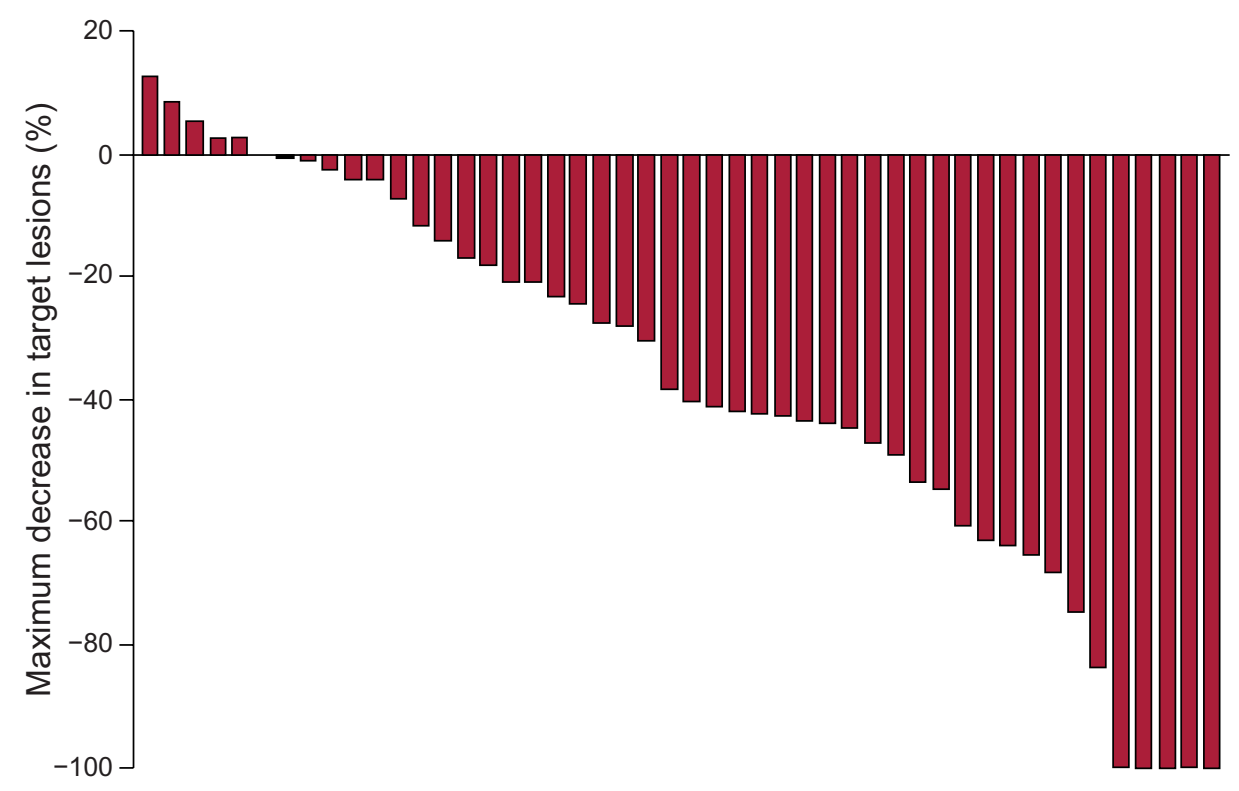

Figure 3 Maximum percentage tumor decrease of target lesions by Response Evaluation Criteria In Solid Tumors.

Notes: Bars represent individual patients. Zero represents baseline (no change); - 100\% represents potential complete response; greater than - $30 \%$ represents potential partial response. Excludes four patients who progressed without post-baseline scans. Reprinted from The Lancet Oncology, Vol 8, Rixe O, Bukowski RM, Michaelson MD, et al, Axitinib treatment in patients with cytokine-refractory metastatic renal-cell cancer: a phase II study, Pages $975-984,{ }^{42}$ Copyright (C) 20I4, with permission from Elsevier.

of 1. Patients in this study were heavily pretreated; in fact, all patients had prior systemic therapy with sorafenib and $22.6 \%$ had also received prior therapy with sunitinib. Approximately two thirds (61\%) of patients had previously received cytokine therapy. The overall response rate was $22.6 \%$, with 14 patients achieving a partial response. Eleven patients $(17.7 \%)$ had stable disease. The clinical benefit rate (complete and partial responses plus stable disease) was $40 \%$. The maximal percentage reduction in target lesions (according to RECIST criteria) during treatment with axitinib was based on dose titration. Some degree of tumor shrinkage with axitinib was seen in 40 of 50 patients $(80 \%)$ for whom post-baseline data were available. Twelve patients without a post-baseline scan were excluded due to study withdrawal (discontinued due to adverse events or withdrawal of consent); these 12 patients were on a starting axitinib dose of $5 \mathrm{mg}$ twice daily. Tumor responses were observed within the group of patients who were dose-titrated to $>5 \mathrm{mg}$ twice daily $(7 / 33 ; 21 \%)$, as well as in patients who remained on $5 \mathrm{mg}$ twice daily or who were dose-modified to $<5 \mathrm{mg}$ twice daily $(7 / 29 ; 24 \%)$. Median progression-free survival was 7.4 months (95\% CI 6.7-11.0) for all patients, with a median follow-up of 22.7 months.

Median progression-free survival was 9.1 months (95\% CI 7.1-11.2) in patients who received axitinib $>10 \mathrm{mg} /$ day and 6.7 months (95\% CI 3.7-7.6) in those who received $\leq 10 \mathrm{mg} /$ day.
These findings suggest that total cross-resistance does not occur between axitinib and sunitinib, sorafenib, and cytokines and that axitinib may have antitumor activity in patients with progressive metastatic RCC.

\section{Phase III efficacy studies}

Following the results of the Phase II studies, the AXIS trial was designed. The Second-Line Axitinib Versus Sorafenib (AXIS) trial is a randomized, open-label Phase III study that evaluated the safety and efficacy of axitinib in patients with progressive metastatic clear-cell RCC who had received one previous line of systemic therapy, including sunitinib, bevacizumab + IFN- $\alpha$, temsirolimus, or cytokine(s), compared with sorafenib. The primary endpoint was progression-free survival as assessed by an independent review committee and defined as time from randomization to either first documentation of RECIST-defined disease progression or death due to any cause; secondary endpoints were overall survival, objective (overall) response rate, and duration of response. The study was designed to detect a $\geq 40 \%$ improvement in median progression-free survival from 5 months with sorafenib, based on available data at that time, to 7 months with axitinib with a power of $90 \%$. The patients were randomized to receive axitinib $5 \mathrm{mg}$ twice daily or sorafenib $400 \mathrm{mg}$ twice daily. For those patients without hypertension or adverse reactions above grade 2 , an increase in axitinib to $7 \mathrm{mg}$ and then $10 \mathrm{mg}$ twice daily was allowed. 
Of 723 subjects randomized, most subjects had received prior sunitinib-based therapy $(54 \%)$ or cytokine-based (interleukin-2 or IFN- $\alpha$ ) therapy (35\%); the remaining subjects (11\%) had received one prior bevacizumab-based or temsirolimus-based therapy. ${ }^{28}$ Patients were assessed for safety at baseline and every 4 weeks thereafter by means of medical history and physical examination, vital signs, clinical laboratory evaluation, and Eastern Cooperative Oncology Group performance status. Safety was assessed throughout the study. Tumor assessments were conducted at screening at weeks 6 and 12, and every 8 weeks thereafter. Patient-reported quality of life was measured using two quality of life instruments, ie, The Functional Assessment of Cancer Therapy Kidney Symptom Index (FKSI) and the FKSI-Disease Related Symptoms (FKSI-DRS) subscale, which specifically measures symptoms associated with advanced RCC.

The AXIS study demonstrated significantly longer median progression-free survival with axitinib versus sorafenib (6.7 versus 4.7 months, respectively, $P<0.0001$ ). In this study, axitinib was superior to sorafenib for the primary endpoint of progression-free survival, with a statistically significant $33 \%(P<0.0001)$ overall reduction of risk of progression or death, with a hazard ratio (HR) of $0.665(95 \%$ CI 0.544-0.812; one-sided $P<0.0001)$. Significant improvements in progression-free survival were also observed in patients treated with sunitinib-based or cytokine-based therapy (Figure 4).

In the AXIS trial, median progression-free survival favored axitinib in patients who had received prior cytokines (12.1 versus 6.5 months; $P<0.0001)$ and those who had received prior sunitinib therapy (4.8 versus 3.4 months; $P=0.0107$ ). In the axitinib arm, axitinib was also observed to perform better for the secondary endpoint of overall response rate. According to the blinded independent review committee, subjects in the axitinib treatment arm had an overall response rate of $19.4 \%$ (95\% CI 15.4-23.9), compared with $9.4 \%$ (95\% CI 6.6-12.9) for those in the sorafenib treatment $\operatorname{arm}(P=0.0001)$.

This is the first randomized Phase III study using an active comparator (sorafenib), which is a VEGF receptor TKI approved for treatment of advanced RCC. The overall survival data were published with a cutoff date of November 1, 2011. The median overall survival was 20.1 months (95\% CI 16.723.4) with axitinib and 19.2 months (17.5-22.3) with sorafenib (HR 0.969, 95\% CI 0.800-1.174; one-sided $P=0.3744) .{ }^{44}$ Updated progression-free survival as assessed by the investigators was also reported. These results are consistent with

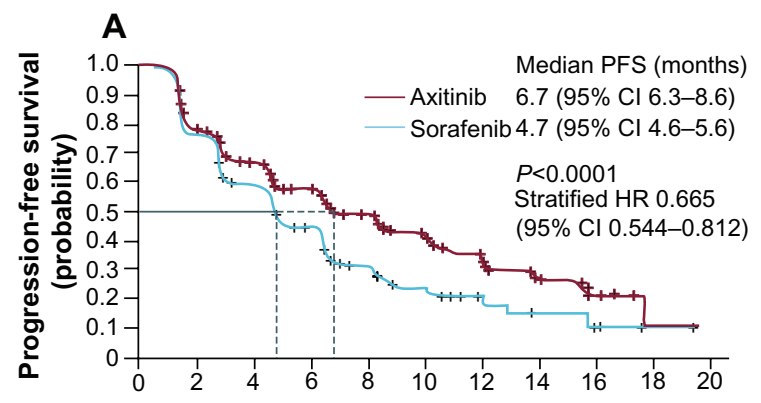

Number at risk

$\begin{array}{rrrrrrrrrrr}\text { Axitinib 361 } & 256 & 202 & 145 & 96 & 64 & 38 & 20 & 10 & 1 & 0 \\ \text { Sorafenib 362 } & 224 & 157 & 100 & 51 & 28 & 12 & 6 & 3 & 1 & 0\end{array}$

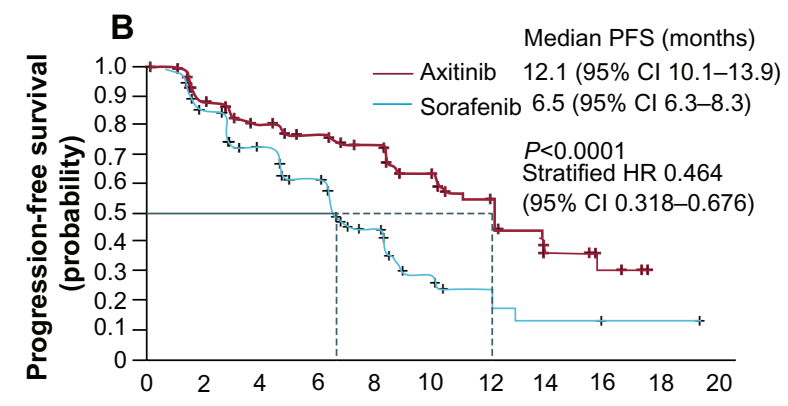

Number at risk

$\begin{array}{lllllllllll}\text { Axitinib } 126 & 98 & 86 & 73 & 55 & 38 & 27 & 10 & 4 & 0 & 0\end{array}$

$\begin{array}{lllllllllll}\text { Sorafenib } 125 & 93 & 75 & 57 & 28 & 12 & 7 & 3 & 2 & 1 & 0\end{array}$

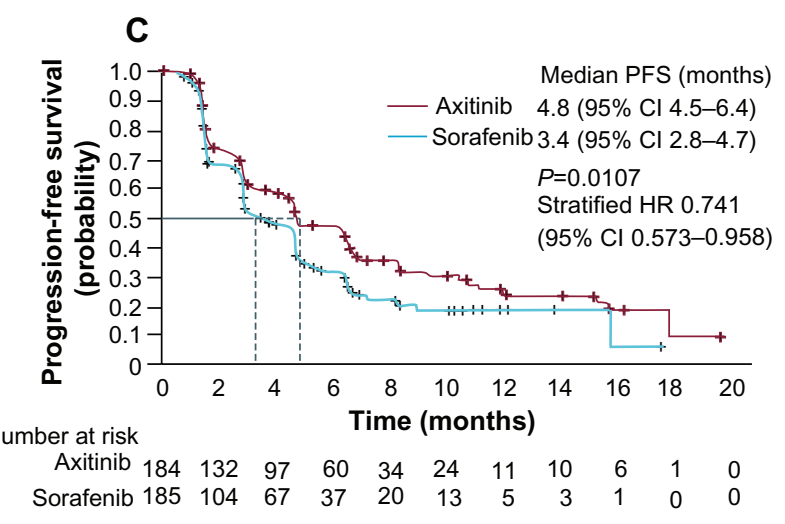

Figure 4 Kaplan-Meier estimated median progression-free survival in patients who received axitinib or sorafenib as second-line therapy for metastatic renal cell cancer. (A) All patients, (B) patients previously treated with cytokine-based regimen, and (C) patients previously treated with sunitinib-based regimen (full analysis set, by independent review committee assessment).

Note: $P$-values based on one-sided, stratified log-rank test. Reprinted from The Lancet, Vol 378, Rini BI, Escudier B, Tomczak P, et al, Comparative effectiveness of axitinib versus sorafenib in advanced renal cell carcinoma (AXIS): a randomised phase 3 trial, Pages 1931-1939, ${ }^{28}$ Copyright @ 201 I, with permission from Elsevier. Abbreviations: $\mathrm{HR}$, hazard ratio; $\mathrm{PFS}$, progression-free survival; $\mathrm{Cl}$, confidence interval.

the investigator-assessed median progression-free survivals previously reported in the initial AXIS publication. ${ }^{28}$ Updated investigator-assessed median progression-free survival was 8.3 months (95\% CI 6.7-9.2) with axitinib and 5.7 months (95\% CI 4.7-6.5) with sorafenib (HR 0.656, 95\% CI 0.552-0.79; one-sided $P<0.0001)$. Updated investigatorassessed median progression-free survival post-sunitinib was 
6.5 months (95\% CI 5.7-7.9) with axitinib and 4.4 months (2.9-4.7) with sorafenib (HR 0.719, 95\% CI 0.572-0.903; one-sided $P=0.0022$ ). The lack of a statistically significant difference in terms of overall survival between axitinib and sorafenib in post-sunitinib patients may have been extensively debated. However, even if overall survival, which was a secondary endpoint for the study, did not differ between the two arms, it is noteworthy that investigator-assessed progressionfree survival in the axitinib group was significantly longer compared with the sorafenib group. The analysis of overall survival might have been confounded by subsequent active treatments which were given to the majority of patients who discontinued study treatment. Furthermore, showing a survival advantage is particularly challenging when survival post progression is long ( $>12$ months), as seen in the AXIS study. Due to the fact that progression-free survival was chosen as the primary endpoint of this study and that active treatments were available beyond progression, the variability introduced by a long survival post progression might have diluted the ability to detect a statistically significant difference in overall survival. ${ }^{45}$ However, it is not possible to rule out that sorafenib may be at least as effective as axitinib in prolonging overall survival.

Additional data for axitinib in first-line therapy are available from AGILE 1046, a Phase II study in which the primary objective was to compare the overall response rate in patients randomized to axitinib + active (axitinib) titration versus axitinib + placebo titration. The overall response rate was $54 \%$ versus $34 \%$, respectively $(P=0.019)$ with a median progression-free survival of 14.6 months. ${ }^{46}$ AGILE 1051, a Phase III study comparing axitinib with sorafenib, failed to meet its ambitious primary endpoint of increasing progression-free survival by $78 \%$ in patients treated with axitinib. This study found no significant difference in median progression-free survival between patients treated with axitinib or sorafenib (10.1 months [95\% CI 7.2-12.1] versus 6.5 months [4.7-8.3], respectively). Although axitinib did not significantly increase progression-free survival in first-line treatment of metastatic RCC compared with sorafenib, it did demonstrate clinical activity and had a valid safety profile ${ }^{47}$ (Table 3). However, this study halted the development of axitinib as first-line therapy for RCC.

At present, AXIS is the only head-to-head randomized clinical trial of axitinib versus sorafenib in patients with metastatic RCC after a first-line therapy, and supports axitinib as second-line therapy for metastatic RCC after failure of prior treatment with sunitinib or a cytokine. Studies conducted with targeted therapies in the second-line setting have indicated better progression-free survival compared with IFN- $\alpha$ and placebo.

TARGET (the Treatment Approaches in Renal Cancer Global Evaluation Trial) compared sorafenib versus placebo in patients pretreated with a cytokine and demonstrated a benefit in terms of progression-free survival in this setting in favor of sorafenib. ${ }^{21}$ Also, the RECORD 1 (Renal Cell cancer treatment with Oral RAD001 given Daily) study compared everolimus and placebo in patients with metastatic RCC which had progressed on VEGF receptor-targeted TKIs. This treatment was not only in the second-line setting, but also in subsequent lines of therapy since $71 \%$ of patients in the everolimus group and $79 \%$ in the placebo group had progressed after more than one previous treatment. Everolimus significantly prolonged progression-free survival compared with placebo (4.9 versus 1.9 months; $P \leq 0.001) .{ }^{13}$ A pivotal study also evaluated pazopanib in treatment-naïve patients pretreated with a cytokine. ${ }^{24}$ Based on these studies, sorafenib and pazopanib are recommended as second-line therapy for patients with metastatic RCC following prior cytokine

Table 3 Treatment-related adverse events in AGILE 1046

\begin{tabular}{|c|c|c|c|c|c|c|}
\hline & \multicolumn{3}{|l|}{ Axitinib $(n=189)$} & \multicolumn{3}{|c|}{ Sorafenib $(n=96)$} \\
\hline & All grades, n (\%) & Grade 3 & Grade 4 & All grades & Grade 3 & Grade 4 \\
\hline Diarrhea & $94(50)$ & $17(9)$ & 0 & $38(40)$ & $5(5)$ & 0 \\
\hline Hypertension & $92(49)$ & $25(13)$ & I & $28(29)$ & I (I) & 0 \\
\hline Weight decrease & $69(37)$ & $16(8)$ & 0 & $23(24)$ & $3(3)$ & 0 \\
\hline Fatigue & $62(37)$ & $9(5)$ & I & $25(26)$ & $\mathrm{I}(\mathrm{I})$ & 0 \\
\hline Decreased appetite & $54(29)$ & $4(2)$ & 0 & $18(19)$ & 0 & 0 \\
\hline PPE & $50(26)$ & $14(7)$ & 0 & $37(39)$ & $15(16)$ & 0 \\
\hline Dysphonia & $44(23)$ & $2(I)$ & 0 & $10(10)$ & 0 & 0 \\
\hline Asthenia & $39(2 \mathrm{I})$ & $13(7)$ & 3 & $15(16)$ & $5(5)$ & 0 \\
\hline Hypothyroidism & $39(2 I)$ & 0 & 0 & $7(7)$ & 0 & 0 \\
\hline Nausea & $37(20)$ & $2(1)$ & 0 & $14(15)$ & $I(I)$ & 0 \\
\hline Rash & $18(10)$ & $2(1)$ & 0 & $19(20)$ & I (I) & 0 \\
\hline
\end{tabular}

Abbreviation: PPE, palmar-plantar erythrodysesthesia. 
therapy because of the robust Phase III evidence for improved progression-free survival with sorafenib and pazopanib compared with placebo in this patient group.

Everolimus is recommended as second-line treatment in patients with VEGF receptor TKI-refractory metastatic RCC because of the significantly improved progression-free survival found in patients refractory to VEGF-targeted therapy (sunitinib and/or sorafenib). The results of the INTORSECT (Investigating Torisel As Second-Line Therapy) study comparing temsirolimus with sorafenib in patients who progressed on treatment with sunitinib at first-line revealed no benefit in progression-free survival for temsirolimus arm. ${ }^{48}$ The TIVO-1 (Tivozanib Versus Sorafenib in first line Advanced RCC) study demonstrated a better progression-free survival of 11.9 months versus 9.1 months for tivozanib versus sorafenib, respectively, in the overall population. In the subgroup of treatment-naïve patients ( $70 \%$ of the overall population) the progression-free survival was 12.7 months versus 9.1 months for tivozanib and sorafenib, respectively. ${ }^{49}$

\section{Safety and tolerability}

The safety profile of axitinib is generally manageable with standard medical intervention. ${ }^{50}$ No unexpected adverse events occurred in the Phase II studies. The most common grade 3 adverse events were hand-foot syndrome, fatigue, hypertension, and diarrhea. In the Phase III trial, some of the grade 3 or higher treatment-related adverse events most frequently reported with axitinib included hypertension (16\%), diarrhea (11\%), fatigue (11\%), and hand-foot syndrome $(16 \%)$, hypertension (11\%), and diarrhea (7\%) in the sorafenib arm. Discontinuation rates due to treatment-related adverse events were $4 \%$ in the axitinib arm and $8 \%$ in the sorafenib arm. ${ }^{28}$

Proactive assessment and management of these adverse events during axitinib therapy can minimize treatment interruptions and ensure optimal benefit from treatment. ${ }^{51,52}$ Recommendations for monitoring, preventing, and treating adverse events can reduce the severity and duration of side effects, as well as improve dose delivery, which may maximize clinical outcomes. Strategies include educating patients about potential adverse events, regular monitoring, and concomitant treatment of adverse events. With these recommendations, the majority of adverse events associated with axitinib can be managed while maintaining patients on therapy. Management of some adverse reactions may require temporary or permanent discontinuation and/or reduction of the axitinib dose. When dose reduction is necessary, according to the pivotal Phase III study, the axitinib dose may be reduced to $3 \mathrm{mg}$ twice daily and further to $2 \mathrm{mg}$ twice daily. Patients who tolerate the axitinib starting dose of $5 \mathrm{mg}$ twice daily with no adverse reactions higher than grade 2 (ie, without severe adverse reactions according to Common Terminology Criteria for Adverse Events version 3.0) for 2 consecutive weeks may have their dose increased to $7 \mathrm{mg}$ twice daily unless blood pressure is $>150 / 90 \mathrm{mmHg}$ or the patient is receiving antihypertensive treatment. Subsequently, using the same criteria, patients who tolerate an axitinib dose of $7 \mathrm{mg}$ twice daily may have their dose increased to a maximum of $10 \mathrm{mg}$ twice daily.

Overall, these results demonstrate that axitinib has clinically meaningful efficacy in subjects with advanced RCC. Efficacy endpoints, including progression-free survival and overall response rate, favored the axitinib treatment arm. The adverse events reported for axitinib were generally manageable by dosing interruption, dose reduction, and/or standard medical therapy.

\section{Patient-reported outcomes}

The AXIS trial also included an evaluation of patient-reported kidney cancer-specific symptoms/functioning and health status as a secondary endpoint. The pivotal AXIS trial evaluated the composite endpoint of time to deterioration (defined as decrease in quality of life), disease progression, and death. More than $90 \%$ of questionnaires were completed during treatment and able to be analyzed for symptom deterioration. The time to deterioration (TTD), assessed by Functional Assessment of Cancer Therapy (FACT)-Kidney Symptom Index-15 (FKSI-15), reported a 17\% risk reduction with axitinib versus sorafenib $(P=0.014)$ and a $16 \%$ risk reduction with axitinib ( $P=0.0203$ ) for TTD evaluated by FKSI-Disease Related Symptoms (FKSI-DRS) ${ }^{28}$ More detailed analysis of secondary endpoints concerning patient-reported outcomes data (kidney cancer-specific symptoms, health status) was done using the Functional Assessment of Cancer Therapy, FKSI, and European Quality of Life questionnaire, 5 dimensions (EQ-5D). The FKSI-15 and EuroQoL (EQ-5D) were completed at screening, after every 4 weeks of therapy, at the end of the study treatment, and at follow-up (28 days after end of therapy). End of treatment and follow-up data were collected in different cycles, reflecting the different times that patients went off treatment. ${ }^{53}$ The patient-reported outcomes were comparable for second-line axitinib and sorafenib, and were maintained at relatively high levels while on treatment, albeit worsened at end of treatment. Because the duration of treatment was longer with axitinib than with sorafenib, time to worsening of symptoms can be delayed longer with axitinib. Given that disease-related symptoms and healthrelated quality of life are better when patients are on treatment 
and progression-free, and axitinib induces significantly longer progression-free survival compared with sorafenib, the patients who receive axitinib are more likely to have longer symptom control that is not compensated by a worsening of patient-reported outcomes. These results also support the treatment with axitinib to delay the worsening of quality of life occurring at the discontinuation of therapy. ${ }^{54}$

\section{Conclusion}

The availability of targeted therapies for the treatment of advanced metastatic RCC has changed the progressionfree survival and overall survival of these patients. To date, seven targeted therapies (sorafenib, sunitinib, bevacizumab/ IFN- $\alpha$ combination, temsirolimus, everolimus, pazopanib, and axitinib) have been investigated and approved by the US Food and Drug Administration and the European Medicines Agency. As second-line treatment, after a first-line cytokinebased therapy, the guidelines of the European Society of Medical Oncology (ESMO) recommend sorafenib [I, A], pazopanib [II, A], and recently axitinib [I, A]. Sunitinib has also demonstrated activity is this setting [III, A]. Based on this premise, VEGF-targeted therapy represents the first-line standard of care for these patients, and the European Society of Medical Oncology guidelines recommend axitinib [I, A] and everolimus [II, A]..$^{55}$

Based on the AXIS results demonstrating significantly longer median progression-free survival with axitinib versus sorafenib (even if no statistically significant differences in overall survival were observed) and on the AGILE results (where no significant difference in median progression-free survival was observed between patients treated with axitinib or sorafenib), axitinib was established as a second-line treatment option for patients with metastatic RCC. In the future, it will be interesting to explore the use of axitinib further in the first-line setting, as well as its role as an adjuvant or neoadjuvant therapy. Predictive factors for efficacy of this molecule should be identified as well.

\section{Disclosure}

GP has served as a consultant for Astellas, Bayer, GSK, and Pfizer. Editorial assistance for the preparation of this manuscript was provided by Luca Giacomelli and Luisa Granzieroon on behalf of Content Ed Net. This assistance was funded by Pfizer. The authors have no other conflicts of interest in this work.

\section{References}

1. Cohen HT, McGovern FJ. Renal-cell carcinoma. $N$ Engl J Med. 2005;353(23):2477-2490.
2. Ferlay J, Autier P, Boniol M, Heanue M, Colombet M, Boyle P. Estimates of the cancer incidence and mortality in Europe in 2006. Ann Oncol. 2007;18(3):581-592.

3. Ferlay J, Parkin D, Steliarova-Foucher E. Estimates of cancer incidence and mortality in Europe in 2008. Eur J Cancer. 2010;46(4):765-781.

4. Linehan WM, Rini BI, Yang JC. Cancer of the kidney. In: De Vita VT, Hellmann S, Rosemberg SA, editors. Cancer, Principles and Practice of Oncology. 9th ed. Philadelphia, PA, USA: Lippincott Williams \& Wilkins, a Wolters Kluwer business; 2011:1161-1182.

5. American Cancer Society. Cancer Facts and Figures. Atlanta, GA, USA: 2013. Available from: http://www.cancer.org/acs/groups/ content/@epidemiologysurveilance/documents/document/acspc028323.pdf. Accessed December 19, 2013.

6. Mattei J, da Silva R, Sehrt D, Molina W, Kim F. Targeted therapy in metastatic renal carcinoma. Cancer Lett. 2013; pii: S0304-3835(13)00707-6.

7. Porta C, Zimatore M, Imarisio I, et al. Gemcitabine and oxaliplatin in the treatment of patients with immunotherapy-resistant advanced renal cell carcinoma: final results of a single-institution Phase II study. Cancer. 2004;100(10):2132-2138.

8. Tamaskar I, Pili R. Update on novel agents in renal cell carcinoma. Expert Rev Anticancer Ther. 2009;9(12):1817-1827.

9. Howlader N, Noone AM, Krapcho M, et al. SEER Cancer Statistics Review, 1975-2010. Bethesda, MD, USA; National Cancer Institute; 2013. Available from: http://seer.cancer.gov/csr/1975_2010/.Accessed December 19, 2013.

10. Negrier S, Escudier B, Lasset C, et al. Recombinant human interleukin-2, recombinant human interferon alfa-2a, or both in metastatic renal-cell carcinoma. Groupe Francais d'Immunotherapie. $N$ Engl J Med. 1998;338(18):1272-1278.

11. Atkins MB, Regan M, McDermott D. Update on the role of interleukin 2 and other cytokines in the treatment of patients with stage IV renal carcinoma. Clin Cancer Res. 2004;10(18 Pt 2):6342S-6346S.

12. Negrier S, Perol D, Ravaud A, et al. Medroxyprogesterone, interferon alfa-2a, interleukin 2, or combination of both cytokines in patients with metastatic renal carcinoma of intermediate prognosis: results of a randomized controlled trial. Cancer. 2007;110(11):2468-2477.

13. Motzer R, Escudier B, Oudard S, et al. Efficacy of everolimus in advanced renal cell carcinoma: a double-blind, randomised, placebocontrolled phase III trial. Lancet. 2008;372(9637):449-456.

14. Molina A, Motzer R. Clinical practice guidelines for the treatment of metastatic renal cell carcinoma: today and tomorrow. Oncologist. 2011;16 Suppl 2:45-50.

15. Molina A, Feldman D, Voss M, et al. Phase 1 trial of everolimus plus sunitinib in patients with metastatic renal cell carcinoma. Cancer. 2012;118(7):1868-1876.

16. Hutson TE. Targeted therapies for the treatment of metastatic renal cell carcinoma: clinical evidence. Oncologist. 2011;16 Suppl 2:14-22.

17. Patard J-J, Pignot G, Escudier B, et al. ICUD-EAU International Consultation on Kidney Cancer 2010: treatment of metastatic disease. Eur Urol. 2011;60(4):684-690.

18. Ljungberg B, Cowan N, Hanbury D, et al. EAU guidelines on renal cell carcinoma: the 2010 update. Eur Urol. 2010;58(3):398-406.

19. Motzer R, Agarwal N, Beard C, et al. NCCN clinical practice guidelines in oncology: kidney cancer. J Natl Compr Canc Netw. 2009;7(6): 618-630.

20. Escudier B, Pluzanska A, Koralewski P, et al. Bevacizumab plus interferon alfa-2a for treatment of metastatic renal cell carcinoma: a randomised, double-blind phase III trial. Lancet. 2007;370(9605):2103-2111.

21. Escudier B, Eisen T, Stadler WM, et al. Sorafenib in advanced clear-cell renal-cell carcinoma. N Engl J Med. 2007;356(2):125-134.

22. Motzer RJ, Hutson TE, Tomczak P, et al. Sunitinib versus interferon alfa in metastatic renal-cell carcinoma. $N$ Engl J Med. 2007;356(2):115-124.

23. Rini BI, Halabi S, Rosenberg JE, et al. Phase III trial of bevacizumab plus interferon alfa versus interferon alfa monotherapy in patients with metastatic renal cell carcinoma: final results of CALGB 90206. J Clin Oncol. 2010;28(13):2137-2143. 
24. Sternberg CN, Davis ID, Mardiak J, et al. Pazopanib in locally advanced or metastatic renal cell carcinoma: results of a randomized phase III trial. J Clin Oncol. 2010;28(6):1061-1068.

25. Hanahan D, Weinberg RA. Hallmarks of cancer: the next generation. Cell. 2011;144(5):646-674.

26. Hudes G, Carducci M, Tomczak P, et al. Temsirolimus, interferon alfa, or both for advanced renal-cell carcinoma. NEngl J Med. 2007;356(22): 2271-2281.

27. Motzer RJ, Escudier B, Oudard S, et al. Phase 3 trial of everolimus for metastatic renal cell carcinoma: final results and analysis of prognostic factors. Cancer. 2010;116(18):4256-4265.

28. Rini BI, Escudier B, Tomczak P, et al. Comparative effectiveness of axitinib versus sorafenib in advanced renal cell carcinoma (AXIS): a randomised phase 3 trial. Lancet. 2011;378(9807):1931-1939.

29. Dabney R, Devine R, Sein N, George B. New agents in renal cell carcinoma. Target Oncol. November 16, 2013. [Epub ahead of print.]

30. Patson B, Cohen RB, Olszanski AJ. Pharmacokinetic evaluation of axitinib. Expert Opin Drug Metab Toxicol. 2012;8(2):259-270.

31. Bukowski R. Third generation tyrosine kinase inhibitors and their development in advanced renal cell carcinoma. Front Oncol. 2012;2:13.

32. Hu-Lowe D, Zou H, Grazzini M, et al. Nonclinical antiangiogenesis and antitumor activities of axitinib (AG-013736), an oral, potent, and selective inhibitor of vascular endothelial growth factor receptor tyrosine kinases 1, 2, 3. Clin Cancer Res. 2008;14(22):7272-7283.

33. Rugo H, Herbst R, Liu G, et al. Phase I trial of the oral antiangiogenesis agent AG-013736 in patients with advanced solid tumors: pharmacokinetic and clinical results. J Clin Oncol. 2005;23(24): 5474-5483.

34. Pithavala Y, Tortorici M, Toh M, et al. Effect of rifampin on the pharmacokinetics of axitinib (AG-013736) in Japanese and Caucasian healthy volunteers. Cancer Chemother Pharmacol. 2010;65(3): 563-570.

35. Poller B, Iusuf D, Sparidans R, Wagenaar E, Beijnen J, Schinkel A. Differential impact of P-glycoprotein (ABCB1) and breast cancer resistance protein (ABCG2) on axitinib brain accumulation and oral plasma pharmacokinetics. Drug Metab Dispos. 2011;39(5): 729-735.

36. Mignogna C, Staibano S, Altieri V, et al. Prognostic significance of multidrug-resistance protein (MDR-1) in renal clear cell carcinomas: a five year follow-up analysis. BMC Cancer. 2006;6:293.

37. Pithavala Y, Tong W, Mount J, et al. Effect of ketoconazole on the pharmacokinetics of axitinib in healthy volunteers. Invest New Drugs. 2012;30(1):273-281.

38. Hoskins JM, Goldberg RM, Qu P, Ibrahim JG, McLeod HL. UGT1A $1 * 28$ genotype and irinotecan-induced neutropenia: dose matters. J Natl Cancer Inst. 2007;99(17):1290-1295.

39. Yap KY, Chui WK, Chan A. Drug interactions between chemotherapeutic regimens and antiepileptics. Clin Ther. 2008;30(8):1385-1407.

40. Kiran P. Typical drug interactions in oncology. US Pharm. 2011; 36(1):7-9.
41. Pithavala YK, Chen Y, Toh M, et al. Evaluation of the effect of food on the pharmacokinetics of axitinib in healthy volunteers. Cancer Chemother Pharmacol. 2012;70(1):103-112.

42. Rixe O, Bukowski RM, Michaelson MD, et al. Axitinib treatment in patients with cytokine-refractory metastatic renal-cell cancer: a phase II study. Lancet Oncol. 2007;8(11):975-984.

43. Rini BI, Wilding G, Hudes G, et al. Phase II study of axitinib in sorafenib-refractory metastatic renal cell carcinoma. J Clin Oncol. 2009;27(27):4462-4468.

44. Motzer RJ, Escudier B, Tomczak P, et al. Axitinib versus sorafenib as second-line treatment for advanced renal cell carcinoma: overall survival analysis and updated results from a randomised phase 3 trial. Lancet Oncol. 2013;14(6):552-562.

45. Amir E, Seruga B, Kwong R, Tannock IF, Ocana A. Poor correlation between progression-free and overall survival in modern clinical trials: are composite endpoints the answer? Eur J Cancer. 2012;48(3): 385-388.

46. Rini BI, Melichar B, Ueda T, et al. Axitinib with or without dose titration for first-line metastatic renal-cell carcinoma: a randomised double-blind phase 2 trial. Lancet Oncol. 2013;14(12):1233-1242.

46. Hutson TE, Lesovoy V, Al-Shukri S, et al. Axitinib versus sorafenib as first-line therapy in patients with metastatic renal-cell carcinoma: a randomised open-label phase 3 trial. Lancet Oncol. 2013;14(13): 1287-1294.

48. Hutson TE, Escudier B, Esteban E, et al. Randomized Phase III trial of temsirolimus versus sorafenib as second-line therapy after sunitinib in patients with metastatic renal cell carcinoma. J Clin Oncol. December 2, 2013. [Epub ahead of print.]

49. Motzer RJ, Nosov D, Eisen T, et al. Tivozanib versus sorafenib as initial targeted therapy for patients with metastatic renal cell carcinoma: results from a phase III trial. J Clin Oncol. 2013;31(30):3791-3799.

50. Escudier B, Gore M. Axitinib for the management of metastatic renal cell carcinoma. Drugs R D. 2011;11(2):113-126.

51. Cohen RB, Oudard S. Antiangiogenic therapy for advanced renal cell carcinoma: management of treatment-related toxicities. Invest $\mathrm{New}$ Drugs. 2012;30(5):2066-2079.

52. Larkin J, Fishman M, Wood L, et al. Axitinib for the treatment of metastatic renal cell carcinoma: recommendations for therapy management to optimize outcomes. Am J Clin Oncol. January 24, 2013. [Epub ahead of print.]

53. Cella D, Escudier B, Rini B, et al. Patient-reported outcomes for axitinib versus sorafenib in metastatic renal cell carcinoma: phase III (AXIS) trial. Br J Cancer. 2013;108(8):1571-1578.

54. Payton S. Kidney cancer: AXIS trial data confirm axitinib as second-line option for mRCC. Nat Rev Urol. 2013;10(6):308.

55. Escudier B, Eisen T, Porta C, et al. Renal cell carcinoma: ESMO Clinical Practice Guidelines for diagnosis, treatment and follow-up. Ann Oncol. 2012;23 Suppl 7:vii65-vii71.
Pharmacogenomics and Personalized Medicine

\section{Publish your work in this journal}

Pharmacogenomics and Personalized Medicine is an international, peerreviewed, open access journal characterizing the influence of genotype on pharmacology leading to the development of personalized treatment programs and individualized drug selection for improved safety, efficacy and sustainability. This journal is indexed on the American Chemical

\section{Dovepress}

Society's Chemical Abstracts Service (CAS). The manuscript management system is completely online and includes a very quick and fair peer-review system, which is all easy to use. Visit http://www.dovepress. com/testimonials.php to read real quotes from published authors. 